\title{
BMC Medical Informatics and Decision Making reviewer acknowledgement 2015
}

Giulia Mangiameli

\section{Contributing reviewers}

The editors of BMC Medical Informatics and Decision Making would like to thank all our reviewers who have contributed to the journal in Volume 15 (2015)

Jos Aarts

Netherlands

Dionisio Acosta

UK

Leila Ahmadian

Iran

Larry Allen

USA

Hussam Alshraideh

Jordan

Milena Anatchkova

USA

Jessica Ancker

USA

Shilo Anders

USA

Giuseppe Andreoni

Italy

Steven Babin

USA

Mirza Baig

New Zealand

Charles Baillie

USA

Gianluca Baio

UK
Erin Bantum

Giulia Barbati

Italy

Laura Barnes

USA

Melissa Baysari

Australia

Jeff Belkora

USA

Brian Bell

UK

Barbara Bennett

Australia

Corinna Bergelt

Germany

Marnie Bertolet

USA

Richard Birtwhistle

Canada

Seiji Bito

Japan

Jennifer Boger

Canada

Carissa Bonner

Australia
Timothy Bonnici

UK

Damian Borbolla

USA

Simone Borsci

UK

Daniel Bossen

Netherlands

Jacques Bouaud

USA

Rick Bradshaw

USA

Olivier Johan Tavai Briët

Switzerland

Benjamin Brown

UK

Angela Buchholz

Germany

Christopher Buckingham

UK

Teresa Burgess

Australia

Harry Burke

USA

Erik Cambria

Singapore

Correspondence: giulia.mangiameli@biomedcentral.com

BioMed Central, Floor 6, 236 Gray's Inn Road, London WC1X 8HB, UK 


\begin{tabular}{|c|c|c|}
\hline $\begin{array}{l}\text { Manuel Campos } \\
\text { Spain }\end{array}$ & $\begin{array}{l}\text { Pascal Coorevits } \\
\text { Belgium }\end{array}$ & $\begin{array}{l}\text { David Dorr } \\
\text { USA }\end{array}$ \\
\hline $\begin{array}{l}\text { Dongsheng Cao } \\
\text { China }\end{array}$ & $\begin{array}{l}\text { Erdal Cosgun } \\
\text { Turkey }\end{array}$ & $\begin{array}{l}\text { Adam Dunn } \\
\text { Australia }\end{array}$ \\
\hline $\begin{array}{l}\text { Fran Carroll } \\
\text { UK }\end{array}$ & $\begin{array}{l}\text { Catherine Craven } \\
\text { USA }\end{array}$ & $\begin{array}{l}\text { Claudio Eccher } \\
\text { Italy }\end{array}$ \\
\hline $\begin{array}{l}\text { German Castellanos-Dominguez } \\
\text { Colombia }\end{array}$ & $\begin{array}{l}\text { Caroline Crawford } \\
\text { USA }\end{array}$ & $\begin{array}{l}\text { Mikel Egana Aranguren } \\
\text { Canada }\end{array}$ \\
\hline $\begin{array}{l}\text { Shaneez Chatharoo } \\
\text { UK }\end{array}$ & $\begin{array}{l}\text { Kathrin Cresswell } \\
\text { UK }\end{array}$ & $\begin{array}{l}\text { Birgit Eiermann } \\
\text { Sweden }\end{array}$ \\
\hline $\begin{array}{l}\text { Niels Chavannes } \\
\text { Netherlands }\end{array}$ & $\begin{array}{l}\text { Kris Cuppens } \\
\text { Belgium }\end{array}$ & $\begin{array}{l}\text { Sylvia Elkhuizen } \\
\text { Netherlands }\end{array}$ \\
\hline $\begin{array}{l}\text { You Chen } \\
\text { USA }\end{array}$ & $\begin{array}{l}\text { Wenrui Dai } \\
\text { China }\end{array}$ & $\begin{array}{l}\text { Mortada El-Shabrawi } \\
\text { Egypt }\end{array}$ \\
\hline $\begin{array}{l}\text { Rie Chiba } \\
\text { Japan }\end{array}$ & $\begin{array}{l}\text { Antonio D'Ambrosio } \\
\text { Italy }\end{array}$ & $\begin{array}{l}\text { Nadiye Erdil } \\
\text { USA }\end{array}$ \\
\hline $\begin{array}{l}\text { Deena Chisolm } \\
\text { USA }\end{array}$ & $\begin{array}{l}\text { Gianfranco Damiani } \\
\text { Italy }\end{array}$ & $\begin{array}{l}\text { Miguel Angel Fernandez Granero } \\
\text { Spain }\end{array}$ \\
\hline $\begin{array}{l}\text { Alan Chiu } \\
\text { USA }\end{array}$ & $\begin{array}{l}\text { Abhijit Dasgupta } \\
\text { USA }\end{array}$ & $\begin{array}{l}\text { Jesualdo Tomas Fernandez-Breis } \\
\text { Spain }\end{array}$ \\
\hline $\begin{array}{l}\text { Songphan Choemprayong } \\
\text { Thailand }\end{array}$ & $\begin{array}{l}\text { Martin Dawes } \\
\text { Canada }\end{array}$ & $\begin{array}{l}\text { Carlos Fernandez-Llatas } \\
\text { Spain }\end{array}$ \\
\hline $\begin{array}{l}\text { Chun-An Chou } \\
\text { USA }\end{array}$ & $\begin{array}{l}\text { Theodore Day } \\
\text { USA }\end{array}$ & $\begin{array}{l}\text { Rama Flarsheim } \\
\text { Canada }\end{array}$ \\
\hline $\begin{array}{l}\text { Yiing-Jenq Chou } \\
\text { Taiwan }\end{array}$ & $\begin{array}{l}\text { Derek De Beurs } \\
\text { Netherlands }\end{array}$ & $\begin{array}{l}\text { Jose F Florez-Arango } \\
\text { Colombia }\end{array}$ \\
\hline $\begin{array}{l}\text { Jean-Paul Chretien } \\
\text { USA }\end{array}$ & $\begin{array}{l}\text { Jeroen De Bruin } \\
\text { Austria }\end{array}$ & $\begin{array}{l}\text { Randi Foraker } \\
\text { USA }\end{array}$ \\
\hline $\begin{array}{l}\text { Katerina Christopoulos } \\
\text { USA }\end{array}$ & $\begin{array}{l}\text { Sarah Deeny } \\
\text { UK }\end{array}$ & $\begin{array}{l}\text { Daniel Fort } \\
\text { USA }\end{array}$ \\
\hline $\begin{array}{l}\text { Theopisti Chrysanthaki } \\
\text { UK }\end{array}$ & $\begin{array}{l}\text { Guilherme Del Fiol } \\
\text { USA }\end{array}$ & $\begin{array}{l}\text { Allison Foster } \\
\text { USA }\end{array}$ \\
\hline $\begin{array}{l}\text { Michele Ciulla } \\
\text { Italy }\end{array}$ & $\begin{array}{l}\text { Spiros Denaxas } \\
\text { UK }\end{array}$ & $\begin{array}{l}\text { Paolo Fraccaro } \\
\text { UK }\end{array}$ \\
\hline $\begin{array}{l}\text { Robyn Clay-Williams } \\
\text { Australia }\end{array}$ & $\begin{array}{l}\text { Don Detmer } \\
\text { USA }\end{array}$ & $\begin{array}{l}\text { Anneke Francke } \\
\text { Netherlands }\end{array}$ \\
\hline $\begin{array}{l}\text { Sean Coady } \\
\text { USA }\end{array}$ & $\begin{array}{l}\text { Haryana Dhillon } \\
\text { Australia }\end{array}$ & $\begin{array}{l}\text { Glaura Franco } \\
\text { Brazil }\end{array}$ \\
\hline $\begin{array}{l}\text { Trevor Cohen } \\
\text { USA }\end{array}$ & $\begin{array}{l}\text { Birthe Dinesen } \\
\text { Denmark }\end{array}$ & $\begin{array}{l}\text { Emma Frew } \\
\text { UK }\end{array}$ \\
\hline $\begin{array}{l}\text { Tiago Colicchio } \\
\text { USA }\end{array}$ & $\begin{array}{l}\text { Brian Dixon } \\
\text { USA }\end{array}$ & $\begin{array}{l}\text { Rocco Friebel } \\
\text { UK }\end{array}$ \\
\hline $\begin{array}{l}\text { Tracy Comans } \\
\text { Australia }\end{array}$ & $\begin{array}{l}\text { Cristian Dogaru } \\
\text { UK }\end{array}$ & $\begin{array}{l}\text { Kevin Fuij } \\
\text { USA }\end{array}$ \\
\hline
\end{tabular}


Marie-Pierre Gagnon

Canada

Xiaohong Gao

UK

Ana García-Bernabeu

Spain

Maria Garcia-Gil

Spain

Jennifer Garvin

USA

Susanne Georgsson Öhman

Sweden

Daniele Giansanti

Italy

Michael Gionfriddo

USA

James Giordano

USA

William Goggins

Hong Kong

Virgilio Gomez Rubio

Spain

William Goossen

Netherlands

Kathleen Gray

Australia

Zachary Grinspan

USA

Stephanie Haas

USA

John Halamka

USA

Ronald Halbert

USA

Andrew Hall

UK

David Hanauer

USA

Ron Leonardus Hubertus Handels

Netherlands

Frank Harrell

USA

Peter Haug

USA
Lotte Haverman

Netherlands

Sarah Hawley

USA

Harald Heinzl

Austria

Michelle Hendriks

Netherlands

Vitaly Herasevich

USA

Antonio Hernández Niñirola

Spain

Patrick Hester

USA

Harry Hochheiser

USA

Jason Hockenberry

USA

Jacob Hofdijk

Netherlands

Bree Holtz

USA

Lars Hölzel

Germany

Peter Hoonakker

USA

Susan Hrisos

UK

William Hsu

USA

Bernice Huang

USA

Ursula Hübner

Germany

J. Marjan Hummel

Netherlands

Raul Igual

Spain

Piper Jackson

Canada

Guoqian Jiang

USA

Constance Johnson

USA
Rachel Johnson

UK

Jaranit Kaewkungwal

Thailand

Charles Kahn

USA

Minna Kaila

Finland

Chandrakar Kamath India

Hong Kang

USA

Garehatty Kanthraj

India

David Kaufman

USA

Karen Kelly-Blake

USA

Ahsan Khandoker

United Arab Emirates

Mehdi Khashei

Iran

Patrick Kierkegaard

UK

Ho Kim South

Korea

Jeffrey Klann

USA

Ela Klecun

UK

Peter Knapp

UK

Peter Kokol

Slovenia

Bevan Koopman

Australia

Georgy Kopanitsa

Russian Federation

Ioannis Korkontzelos

UK

Grigorios Kotronoulas

UK

Markus Kreuzthaler

Austria 
Levente Kriston

Germany

Anand Kulanthaivel

USA

Craig Kuziemsky

Canada

Hallvard Laerum

Norway

Uttama Lahiri

India

Pauline Siew Mei Lai

Malaysia

Douglas Lake

USA

Deirdre Lane

UK

Annie Lau

Australia

Yvon Lebranchu

France

Tian-Fu Lee

Taiwan

Young Ji Lee

USA

France Légaré

Canada

Pascale Lehoux

Canada

Lasse Lensu

Finland

Hugo Leroux

Australia

M. Leu

USA

Jörg D. Leuppi

Switzerland

Haomin Li

China

Linda Li

Canada

Wenmin Li

China

Yong Li

China
Zhongjie Li

China

Valentina Lichtner

UK

Sarah Lillie

USA

Ellen Lipstein

USA

Nan Liu

Singapore

Zengjian Liu

China

Miguel López-Coronado

Spain

Karen Lorimer

UK

Jake Luo

USA

Yashar Maali

Australia

Giovanni Maccioni

Italy

Lynette Mackenzie

Australia

Nicola Mackintosh

UK

Vijay Mago

Canada

Jose Alberto Maldonado Spain

Ben Marafino

USA

Mar Marcos

Spain

Chris Martin

UK

Catalina Martínez

Costa Austria

Barbara Massoudi

USA

J. Mac Mccullough

USA

Michelle Mcdowell

Germany
Brian Mckinstry

UK

Amaia Mendez Zorrilla

Spain

Jane Messina

USA

Margreet Michel-Verkerke

Netherlands

Armin R. Mikler

USA

Laura Militello

USA

Dragana Miljkovic

Slovenia

Anne Miller

Australia

Victor Minichiello

Australia

Riccardo Miotto

USA

Jose Joaquin Mira

Spain

Alin Moldoveanu

Romania

Linda Moniz

USA

Roger Morbey

UK

Sandra Morelli

Italy

Hamid Mukhtar

Saudi Arabia

Adolfo Muñoz Carrero

Spain

Radhakrishnan Nagarajan USA

Kim Nazi

USA

Aurelie Neveol

France

Axel Newe

Germany

Huong Nguyen

USA 
Stuart Nicholls

Canada

Edward Nicol

South Africa

Mehrbakhsh Nilashi

Malaysia

Angelo Nuzzo

Austria

Mary Ann O'Brien

Canada

William Ogallo

Kenya

Hans Ossebaard

Netherlands

Casey Overby

USA

Zenggang Pan

USA

Chris Paton

UK

Marco Paulli

ITALY

Philip Payne

USA

Leandro Pecchia

UK

Linda Peelen

Netherlands

Robert Penfold

USA

David Perez-Rey

Spain

Lionel Perrier

France

Rimma Pivovarov

USA

Petra Povalej Brzan

Slovenia

John Powell

UK

Mattia Prosperi

USA

Siyu Qian

Australia
Preethi Raghavan

USA

Rebecca Randell

UK

Luke Rasmussen

USA

Raj Ratwani

USA

Pradeep Ray

Australia

Rachel Richesson

USA

Britta Ricker

USA

Greg Roberts

Australia

Kirk Roberts

USA

Álvaro Rocha

Portugal

Pedro Pereira Rodrigues

Portugal

Lionel Rostaing

France

Robert Rudin

USA

Pekka Ruotsalainen

Finland

Mandy Ryan

UK

Lucia Sacchi

Italy

Satya Sahoo

USA

Farrant Sakaguchi

USA

Frank Sandmann

UK

Reidun Sandvik

Norway

Silvina Santana

Portugal

Nicola Saplding

UK
Philip Scott

UK

Karen Sepucha

USA

Walter Sermeus

Belgium

Berkan Sesen

UK

Aviv Shachak

Canada

Nigam Shah

USA

Megan Shen

USA

Soo-Yong Shin

South Korea

Mauro Silvestrini

Italy

Sonal Singh

USA

Peter Singleton

UK

Sam Smith

UK

Parikshit Sondhi

USA

Joao Sousa

Portugal

Ross Sparks

Australia

Matthew Sperrin

UK

Ton Spil

Netherlands

Stephen Andrew Spooner USA

Cord Spreckelsen

Germany

Douglas Steinke

UK

Gregor Stiglic

Slovenia

Ellen Stuart

Ireland 
Abdülhamit Subaşı

Bosnia and Herzegovina

Xing-Guo Sun

China

Michael Swash

UK

Stephen Swift

UK

Ricky Taira

USA

Anhui Tan

China

Derek Tang

USA

Huibert Tange

Netherlands

Cui Tao

USA

Alan Taylor

Australia

Darlene Taylor

Canada

Johanna Taylor

UK

Richard Thomson

UK

Thankam Thyvalikakath

USA

Binyam Tilahun

Ethiopia

Salvador Tortajada

Spain

Monica Tremblay

USA

Athanasios Tsanas

UK

Samson Tu

USA

Francesco Turturro

USA

Alexandros Tzallas

Greece

Rupa Valdez

USA
Vanya Van Belle

Belgium

Peter Van Der Putten

Netherlands

Heleen Van Der Sijs

Netherlands

Mariette Van Engen-Verheul

Netherlands

Tjeerd Van Staa

UK

Gonzalo Vazquez-Prokopec

USA

Aman Verma

Canada

Karin Verspoor

Australia

Susanne Vijverberg

Netherlands

David Vivas-Consuelo

Spain

Silke Von Esenwein

USA

Frans Voorbraak

Netherlands

Victoria Anne Wade

Australia

Fei Wang

USA

Jing Wang

USA

Jeremy Warner

USA

Marieke Weernink

Netherlands

Charlene Weir

USA

James Weisfeld-Adams

USA

Jobke Wentzel

Netherlands

Perla Werner

Israel

Susan White

USA
James Whyte

USA

Michael Wiegand

Germany

Jane Wilcox

UK

Chris Williams

UK

Qi Xie

China

Lei Yang

China

Po-Yin Yen

USA

Meliha Yetişgen

USA

Tracey Young

UK

Ping Yu

Australia

Konstantinos Zacharias

UK

Fengqing Zhang

USA

Kui Zhang

USA

Pei Zhang

USA

Ping Zhang

USA

Rui Zhang

USA

Wei Zhang

USA

Zhen Zhang

USA

Guoru Zhao

China

Wojciech Ziarko

Canada

Jördis Maria Zill

Germany

Kate Zinszer

Canada 